Original Paper http://ajol.info/index.php/ijbcs http://indexmedicus.afro.who.int

\title{
Recettes ethno-vétérinaire à base de plantes médicinales utilisées pour le traitement de la fièvre aphteuse au Bénin
}

\author{
E.M.B. HOUNDJE, C.A. Ogni, N. NOUDEKE, S. FAROUGOU, A.K.I. YOUSSAO* et \\ T.M. KPODEKON
}

Université d'Abomey-Calavi (UAC), Ecole Polytechnique d'Abomey-Calavi (EPAC), Département de Production et Santé Animales, Laboratoire de Recherches en Biologie Appliquée (LARBA), 01 BP 2009 Cotonou, Bénin.

*Auteur correspondant ; E-mail : iyoussao@yahoo.fr, Tél : (+229) 97912074.

\section{RESUME}

L'usage des plantes dans le traitement des pathologies constitue une pratique courante chez les éleveurs au Bénin. La présente étude a pour but de répertorier les recettes utilisées par les éleveurs pour traiter la fièvre aphteuse. L'enquête s'est déroulée auprès de 370 éleveurs diversement répartis dans onze départements du Bénin, entre juillet et décembre 2015. Elle révèle que pour traiter la fièvre aphteuse, la quasi-totalité des éleveurs enquêtés associent les recettes à base de plantes médicinales aux soins vétérinaires. Au total, 32 types de recettes utilisant 32 espèces de plantes ont été récapitulés chez les éleveurs. Vitellaria paradoxa est l'espèce de plante la plus citée dans 3 types de recettes. Les espèces de plante telles Citrus limon L., Gossypium arboreum L., Pterocarpus erinaceus, Sorghum bicolor L., Acacia nilotica L., Lannea acida, Khaya senegalensis, Agelanthus dodoneifolius ont été chacune impliquées dans 2 recettes. Les écorces sont impliquées dans 14 types de recettes et la macération constitue la méthode la plus utilisée avec $27 \%$ de types de recettes suivis de la poudre avec $24 \%$. Les soins vétérinaires sont basés sur l'utilisation des antibiotiques (Péniprocaïne $^{\circledR}$ ou l'Oxytétracycline ${ }^{\circledR}$ ). D'autres études pourront révéler l'efficacité de ces plantes.

(C) 2016 International Formulae Group. All rights reserved.

Mots clés : Pathologie, aphtes, recettes endogènes, phytopharmacologie, bovin.

\section{Ethno-veterinary recipes of medicinal plants using for the treatment of foot and mouth disease in Benin}

\begin{abstract}
Foot and mouth disease (FMD) is a highly contagious viral disease of even-toed ungulated and is endemic in most of African countries. A survey using semi-structured questionnaires was undertaken to 370 breeders of the eleven husbandry departments in Benin to identify the recipes used by breeders to treat cases of FMD. A total of 32 types of recipes using 32 medicinal plants were listed by breeders. Vitellaria paradoxa is the most cited with three types of recipes. Thus medicinal plants such as Citrus limon L., Gossypium arboretum
\end{abstract}


L., Pterocarpus erinaceus, Sorghum bicolor L., Acacia nilotica L., Lannea acida, Khaya senegalensis, Agelanthus dodoneifolius were each involved in two types of recipes. Barks are involved in 14 types of recipes and maceration is the method most used with $27 \%$ types of recipes followed by the powder with $24 \%$. Animal health care is based on the use of antibiotics such as Peni-procaïne ${ }^{\circledR}$ or Oxytétracycline ${ }^{\circledR}$. This study is providing indigenous resources using to treat foot and mouth disease in Benin. Others studies could reveal which one are best.

(C) 2016 International Formulae Group. All rights reserved.

Keywords: Pathology, ulcers, endogenous recipes, phytopharmacology, cattle.

\section{INTRODUCTION}

La fièvre aphteuse (FA) est une maladie virale qui affecte les mammifères artiodactyles domestiques et sauvages. Elle est due à un virus de la famille des Picornaviridae, du genre Aphthovirus. Elle se manifeste par la fièvre, une salivation intense, une grande morbidité, une mortalité faible chez les adultes et élevée chez les veaux et l'apparition de vésicules au niveau de l'épiderme de la muqueuse buccale, des espaces inter-digitées et du bourrelet coronaire (Grubman et Baxt, 2004). En raison de ses intérêts économique (chute de la production $\mathrm{du}$ lait et perte en viande) et médical, cette maladie fait aujourd'hui l'objet de plusieurs études à travers le monde. En Afrique, les stratégies de lutte et de contrôle de la fièvre aphteuse varient d'une région à une autre (Gorna et al., 2014) et dépendent du système d'élevage et des plans nationaux et régionaux adoptés. Les pays indemnes du virus sans vaccination tels que le Madagascar, l'Ile Maurice, les Seychelles, les pays de la Communauté de développement d'Afrique Australe (SADC) ainsi que certains pays d'Afrique de l'Est, ont adopté la stratégie de zones indemnes sans vaccin pour accéder aux commerces internationales de bétail (Rweyemamu et al., 2008). Par contre, l'Afrique de l'Ouest, prédominée par le pastoralisme caractérisé par la transhumance des animaux sur de longues distances pour des raisons d'alimentation ou de commerce, est à l'étape de caractérisation du virus et d'essai de vaccination (Fasina et al., 2013).
Le Bénin est l'un des pays d'Afrique de l'Ouest à l'étape d'élaboration de vaccins. Il est frontalier au Nigéria, au Togo, au Niger et au Burkina-Faso. Ces deux derniers pays s'ouvrent, à travers le commerce international de bétail, sur les pays d'élevage de bovins tels que le Mali, le Sénégal et les pays de l'Afrique du Nord.

En raison de cette position géographique et de son climat, le Bénin reçoit tout au long de l'année et presque sur toute l'étendue du territoire national, des troupeaux des pays limitrophes (DED, 2006). Maladie transfrontalière, la fièvre aphteuse sévit de manière enzootique au Bénin. Elle constitue une priorité pour les éleveurs en raison des pertes économiques pouvant aller jusqu'à 50\% de la valeur marchande de l'animal (CouacyHymann et al., 2006). En l'absence d'un programme national de vaccination, et par attachement à la tradition, les éleveurs font généralement recours à l'utilisation de recettes à base de plantes médicinales pour traiter ou atténuer l'effet de la maladie sur les animaux.

En Afrique et particulièrement au Bénin, plusieurs travaux de recherches rapportent aujourd'hui le regain d'intérêt de l'utilisation des plantes médicinales par les éleveurs pour traiter leurs animaux. Ainsi, Toyang et al. (2007) ont fait une synthèse sur des traitements par l'ethnomédecine de quelques pathologies animales en Afrique subsaharienne. D'autres auteurs ont travaillé de façon spécifique sur certains types de pathologies au Bénin. Il s'agit de Akouedegni et al. (2012) sur les plantes galactogènes, Ogni 
et al. (2014) sur les plantes utilisées pour traiter les pathologies parasitaires, Dassou et al. (2014) sur la diversité de ces plantes, Ogni et al. (2016) sur les plantes utilisées dans le traitement des pathologies d'origine bactérienne et Kpodékon et al. (2015a) sur les plantes utilisées pour le contrôle des maladies à incidence négative sur la production du lait des vaches. Ce regain d'intérêt sur l'usage des plantes médicinales au Bénin se justifie par l'indisponibilité des médicaments au niveau des éleveurs, la fréquente résistance des animaux aux produits vétérinaires et par le coût élevé des produits empêchant ainsi les éleveurs qui disposent d'un faible revenu pour accéder.

Pour une meilleure valorisation des plantes médicinales, il est alors important d'établir une liste exhaustive des plantes spécifiques à chaque pathologie animale en vue de remédier aux problèmes de santé animale. Cette liste associée à des études de l'efficacité de ces plantes sur cette pathologie indexée permettrait de pallier aux problèmes de santé des animaux surtout dans les zones où les soins réglementaires sont inexistants, irréguliers ou coûteux.

La présente étude a pour but, dans un contexte d'absence de stratégie de contrôle de la fièvre aphteuse au Bénin, de i) faire un inventaire des plantes utilisées par les éleveurs dans le traitement de cette pathologie; ii) décrire les modes de préparation et d'utilisation ainsi que les parties de plantes incorporées dans les recettes.

\section{MATERIEL ET METHODES}

\section{Zone d'enquête}

La présente étude a été réalisée dans les départements de l'Atacora, de l'Alibori, du Borgou, de la Donga, des Collines, du Zou, de l'Ouémé, du Plateau, du Couffo, du Mono et de l'Atlantique, situés sur le territoire du Bénin. Situé entre $6^{\circ} 15^{\prime}$ et $12^{\circ} 25^{\prime}$ de latitude nord et $0^{\circ} 40^{\prime}$ et $3^{\circ} 45^{\prime}$ de longitude est, le Bénin est caractérisé par 3 zones climatiques (subéquatoriale au sud, soudano-guinéenne au centre et soudanien au nord), un réseau fluviale assez riche et une végétation presque permanente (Figure 1).

$\mathrm{Au}$ Nord du pays, il existe les départements de l'Atacora, de l'Alibori, du Borgou et de la Donga. L'Atacora représente le château d'eau car il est la source des grands fleuves du Bénin et du Togo. Il héberge la réserve de la Pendjari ainsi que les forêts classées de l'Alibori supérieur. La végétation dans le Nord est dominée par des espèces telles que Acacia seyal, Acacia sieberiana, Parkia biglobosa (néré) Butyrospermun paradoxa (karité) et Adansonia digitata (baobab) qui sont des essences protégées. On observe également dans cette partie du Bénin des forêts peuplées d'essences telles que le caïlcédrat (Khaya Senegalensis), le faux acajou (Khaya grandifolia), et le lingué (Afzélia africana), Hyparhénia sp, Loudetia $\mathrm{sp}$, Andropogon sp, Pennisetum sp et de nombreuses Papillionacées, Rubiacées etc... (INSAE, 2004). Dans le département de la Donga, la végétation est dense et constituée de forêts galeries. Les forêts classées occupent plus de la moitié de la commune de Bassila et une partie de la commune de Djougou. Le karité et le Néré sont des espèces qui prédominent dans ce département. La végétation dans le département du Borgou est luxuriante et se raréfie au fur et à mesure que l'on avance vers l'Alibori. Elle est caractérisée par une savane diversifiée sous 4 formes. Il s'agit des savanes herbeuses, arborées, boisées et de la forêt galerie.

$\mathrm{Au}$ Centre du Bénin, il y a les départements des Collines et du Zou. Dans le département des Collines, la savane arborée à Daniella Oliveiri est la végétation dominante et est beaucoup plus prononcée vers le Nord. Les essences les plus répandues de nos jours sont le karité, le néré et le caïlcédrat. Par contre, l'iroko a presque disparu (INSAE, 2004). 
Le sud du pays comprend les départements du Couffo et du Mono à l'Ouest, du Plateaux et de l'Ouémé à l'Est, de l'Atlantique et du Littoral au Sud. La végétation est dominée par le palmier à huile et les cultures vivrières. Toutefois, il y a encore quelques forêts galeries (INSAE, 2004).

\section{Matériel}

Le matériel utilisé au cours de l'étude a été constitué de fiches d'enquêtes qui renseignent sur le profil des éleveurs, les signes associés à la reconnaissance de la maladie, les périodes d'apparition des maladies, les soins pratiqués, les recettes utilisées ainsi que les motivations des éleveurs par rapport au choix de l'usage des plantes; d'appareil photo numérique et de l'Herbier National du Bénin.

\section{Méthodes d'enquête}

Les données ont été collectées sur la base d'un questionnaire adressé à 370 éleveurs diversement répartis dans les onze départements ciblés. Dans chacun des quatre départements du Nord, 40 éleveurs ont été sélectionnés contre 30 dans chaque département du Sud. L'enquête s'est déroulée entre juillet et décembre 2015. La sélection des éleveurs a été faite sur la base de leur disponibilité et de leur habitude à travailler avec les agents d'élevage. La méthodologie utilisée pour la collecte des données est celle de l'enquête rétrospective qui fait appel à la mémoire de l'éleveur. L'enquête s'est déroulée sur la base d'entretien semi-direct au niveau des marchés à bétail pour le questionnaire et dans les aires d'élevages pour prendre les photos des espèces végétales indiquées. Le choix des marchés à bétail se justifie par le fait qu'ils permettent le regroupement d'éleveurs de tout âge et parfois de diverses ethnies. Cela à l'avantage d'obtenir le nom des espèces végétales en langue locale ainsi que les différentes utilisations liées aux expériences des enquêtés. Pour faciliter l'identification des plantes indexées, des herbiers ont été réalisés in situ et des photographies ont été effectuées.

\section{Analyse statistique}

Les fiches d'enquête ont été dépouillées et codées dans le tableur Excel. A partir des tableaux croisés dynamiques, des tableaux de contingence ont été édités et exportés dans le logiciel Stata IC 11. La fréquence de citation $(\mathrm{Fc})$ des plantes par département a été calculée selon la formule :

$F c=\frac{\text { Nombre de citations par plante }}{\text { Nombre total de citations de toutes les plantes }}$

\section{RESULTATS}

\section{Caractéristiques socio-professionnelles des enquêtés}

L'enquête sur les recettes endogènes utilisées dans le contrôle de la fièvre aphteuse au Bénin a révélé que la quasi-totalité des éleveurs enquêtés est de sexe masculin et répartis en quatre classes d'âges : $0-29$ ans $(0,54 \%), 30-49$ ans $(28,65 \%), 50-59$ ans (38,38\%), 60-69 ans $(25,40 \%)$ et plus de 70 ans $(7,03 \%)$ (Figure 2). Les éleveurs enquêtés sont majoritairement d'ethnie peulh $(71,35 \%)$, suivis des dendi $(14,32 \%)$, des yorouba $(14,32 \%)$ et des bariba $(3,24 \%)$ avec des proportions respectives de 14,32\% (Figure 3).

A l'exception du département de l'Ouémé, les éleveurs non instruits sont les plus nombreux dans les autres départements, suivis respectivement des éleveurs de niveaux primaire et secondaire (Figure 4). Les éleveurs de niveau universitaire sont minoritaires et les agro-éleveurs ont été seulement rencontrés dans les départements de l'Alibori et de la Donga. Au cours de l'enquête, les individus de niveau primaire ont été plus rencontrés dans le Département de l'Ouémé. 
Perception des éleveurs sur l'identification et les périodes favorables d'apparition de la fièvre aphteuse

Dans tous les départements, les éleveurs ont reconnu la fièvre aphteuse à travers les signes cliniques observés (Figure 5). Ainsi, un premier groupe constitué de $46 \%$ des éleveurs reconnais la maladie par l'apparition des plaies au niveau de la bouche et des trayons. Le deuxième groupe constitué de $43 \%$ des éleveurs identifie la maladie par les boiteries et l'inappétence des animaux atteints. Cependant, $11 \%$ des éleveurs reconnaissent la maladie à travers la totalité des signes cliniques suscités.

Les périodes favorables d'apparition indiquées par les éleveurs pour cette pathologie varient d'une zone à une autre. Au total, 4 zones ont été retenues. Dans la première zone regroupant les départements de l'Atacora et de l'Alibori, la plupart des éleveurs $(93,2 \%)$ indiquent la présence de la fièvre aphteuse entre juin et août puis en fin novembre. Dans la zone 2 constituée des départements de Borgou et de la Donga, elle apparait entre mars et juillet, puis entre novembre à janvier. Quant à la zone 3 prenant en compte les départements des Collines, du Zou et du Couffo, elle s'observe tout au long de l'année. Enfin, dans la quatrième zone constituée de l'Ouémé, du Plateau, de l'Atlantique et du Mono, la fièvre aphteuse s'observe surtout en saison sèche entre décembre et mars.

\section{Motivations des éleveurs ayant recours à la médecine vétérinaire traditionnelle}

L'enquête révèle que tous les éleveurs ont recours aux soins vétérinaires pour traiter leurs animaux contre la fièvre aphteuse. Les principaux médicaments utilisés sont essentiellement des antibiotiques (Péniprocaïne $^{\circledR}$, Péni-streptomycine $^{\circledR}$, Oxycline $^{\circledR}$, Sulfa33 $^{\circledR}, \quad$ Oxytétracycline $^{\circledR}, \quad$ Vétotrix $^{\circledR}$, Vétospray) ${ }^{\circledR}$. L'appui du vétérinaire vient le plus souvent lorsque les plaies se surinfectent en raison du mode d'élevage (déplacement continu). Les raisons évoquées en faveur de l'usage des recettes endogènes dans le traitement de la fièvre aphteuse sont l'absence ou le retard du vétérinaire à intervenir, l'inefficacité de certains traitements et l'absence de programme de vaccination contre la fièvre aphteuse au Bénin. Ces mêmes éleveurs utilisent les recettes ethnovétérinaires pour leurs animaux, avant et après les soins vétérinaires.

\section{Recettes à base de plantes médicinales utilisées pour le traitement de la fièvre aphteuse au Bénin}

$\mathrm{Au}$ total, 32 espèces de plantes ont été identifiées dans les 32 types de recettes retenues par les éleveurs (Tableau 1). Les parties de plantes les plus utilisées sont les écorces impliquées dans $36 \%$ de types de recettes, suivies des feuilles retrouvées dans $23 \%$. Les racines ont été utilisées dans $13 \%$ de types de recettes et les fruits dans $10 \%$ (Figure 6). La macération est la méthode la plus utilisée $(27 \%)$ pour préparer ces différents types de recettes. Les autres méthodes de préparation des plantes utilisées pour le traitement de la fièvre aphteuse sont par ordre d'importance la poudre $(24 \%)$, la décoction $(18 \%)$, la trituration $(12 \%)$, la fumigation $(9 \%)$, l'infusion $(3 \%)$, le frottage $(3 \%)$ et la calcination (3\%) (Figure 7). Divers ingrédients sont ajoutés tel que le sel de cuisine ou la potasse lors des préparations. Il ressort également des résultats de l'enquête que Vitellaria paradoxa est l'espèce de plante la plus citée dans 3 types de recettes. Les espèces de plante telles Citrus limon L., Gossypium arboreum L., Pterocarpus erinaceus, Sorghum bicolor L., Acacia nilotica L., Lannea acida, Khaya senegalensis, Agelanthus dodoneifolius ont été chacune impliquées dans 2 types de recettes (Figure 8). 


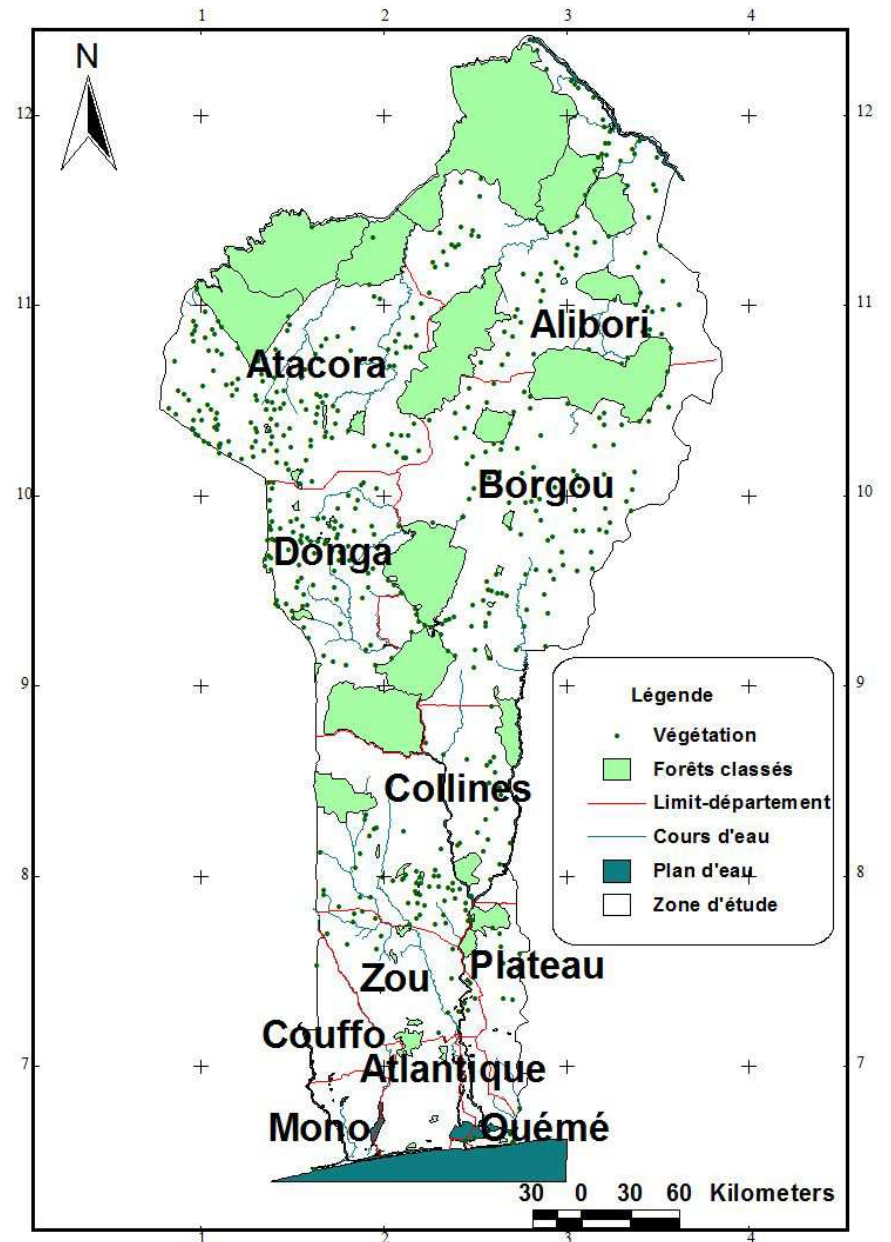

Figure 1 : Carte montrant les départements de l'étude au Bénin.

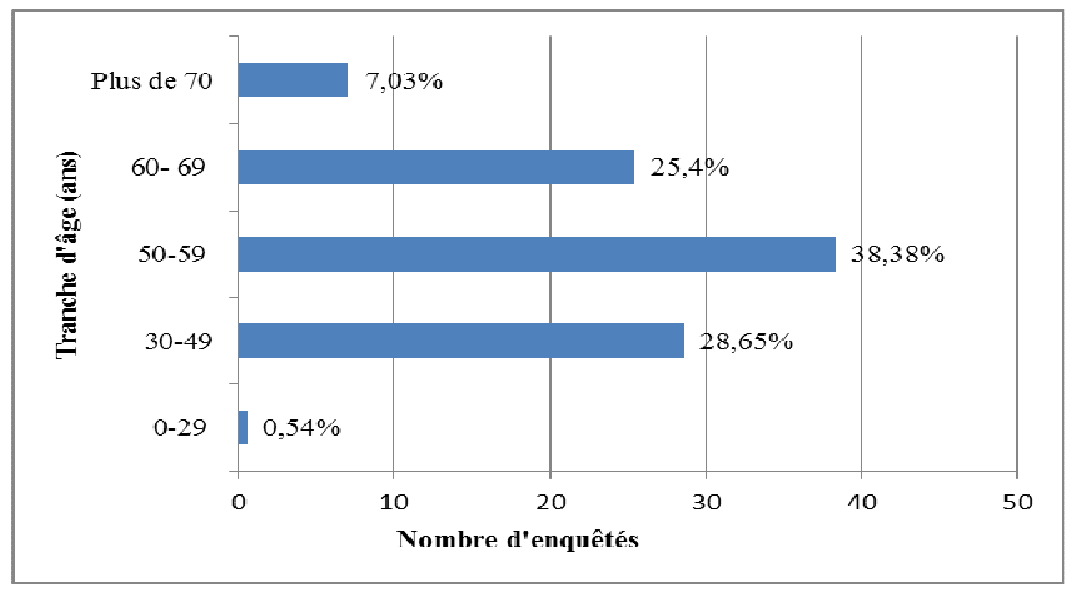

Figure 2 : Distribution des éleveurs suivant les classes d'âge. 


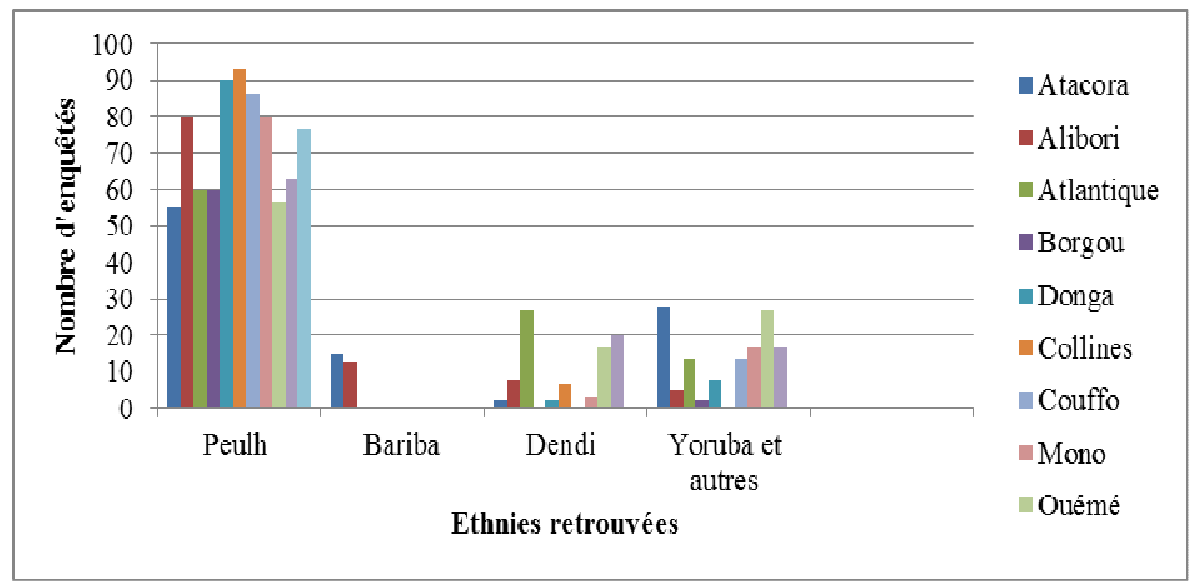

Figure 3 : Fréquences ethniques des enquêtés par département.

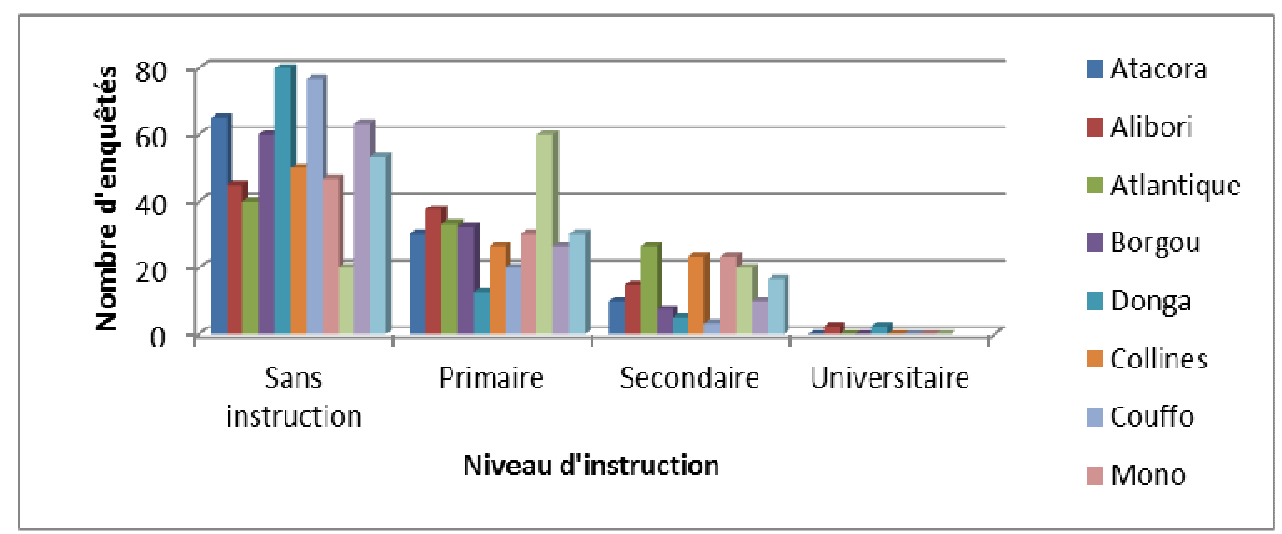

Figure 4 : Niveau d'instruction des éleveurs par département.

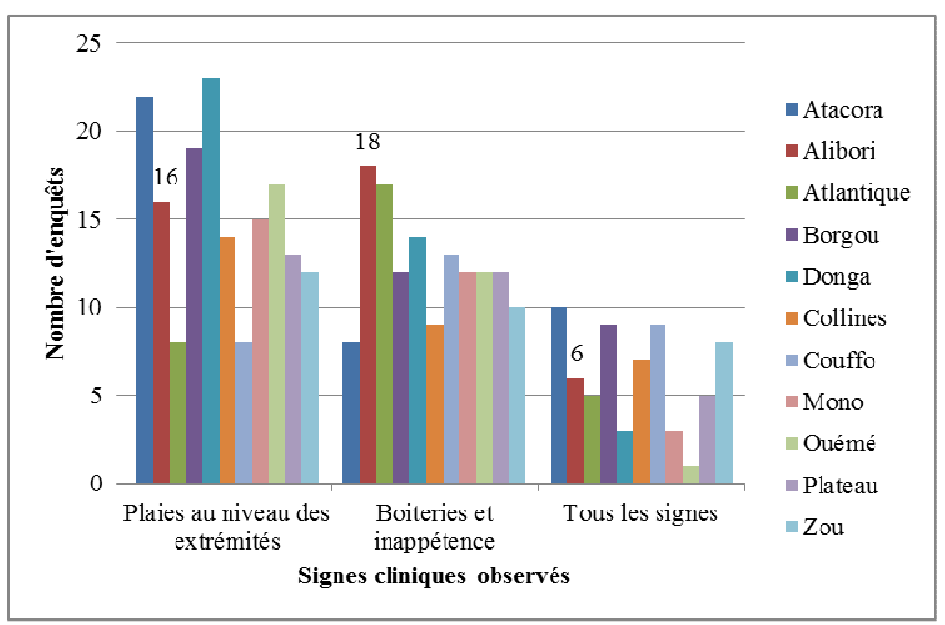

Figure 5 : Fréquence des enquêtés suivant les signes d'identification de la fièvre aphteuse. 


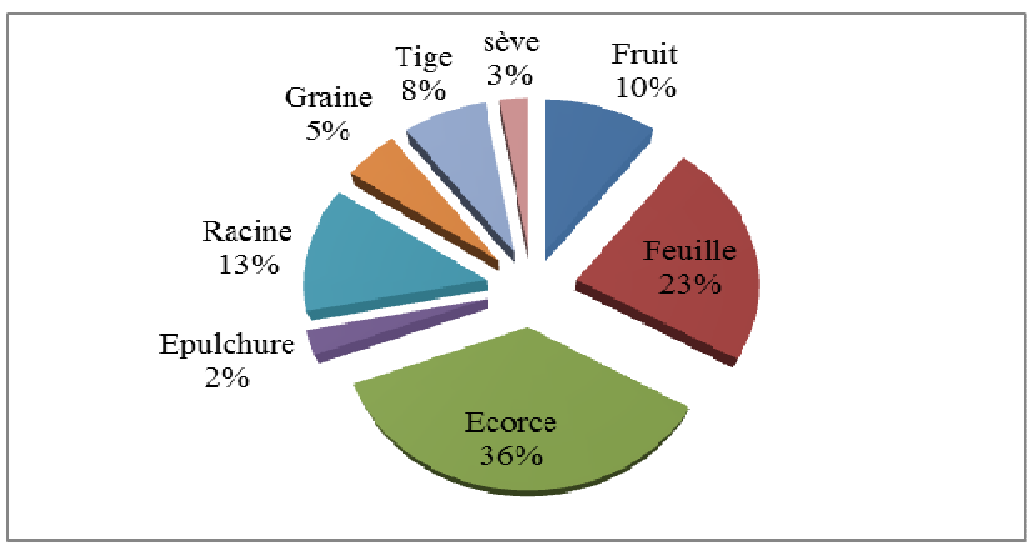

Figure 6 : Proportion des parties d'organe de plantes utilisées dans la composition des recettes.

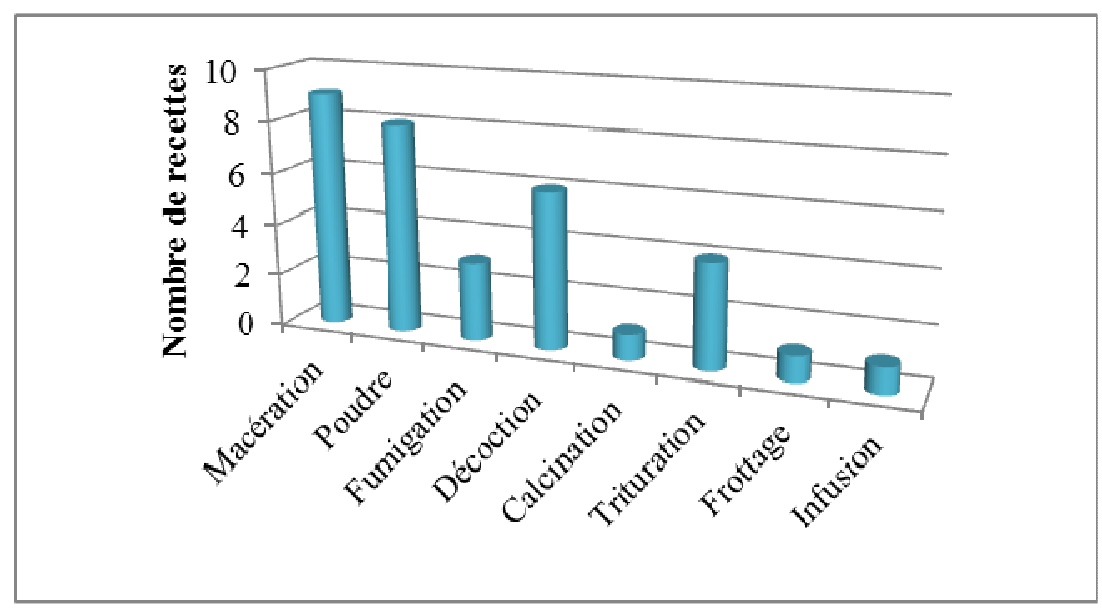

Figure 7 : Méthodes de préparation des recettes utilisées pour le contrôle de la fièvre aphteuse.

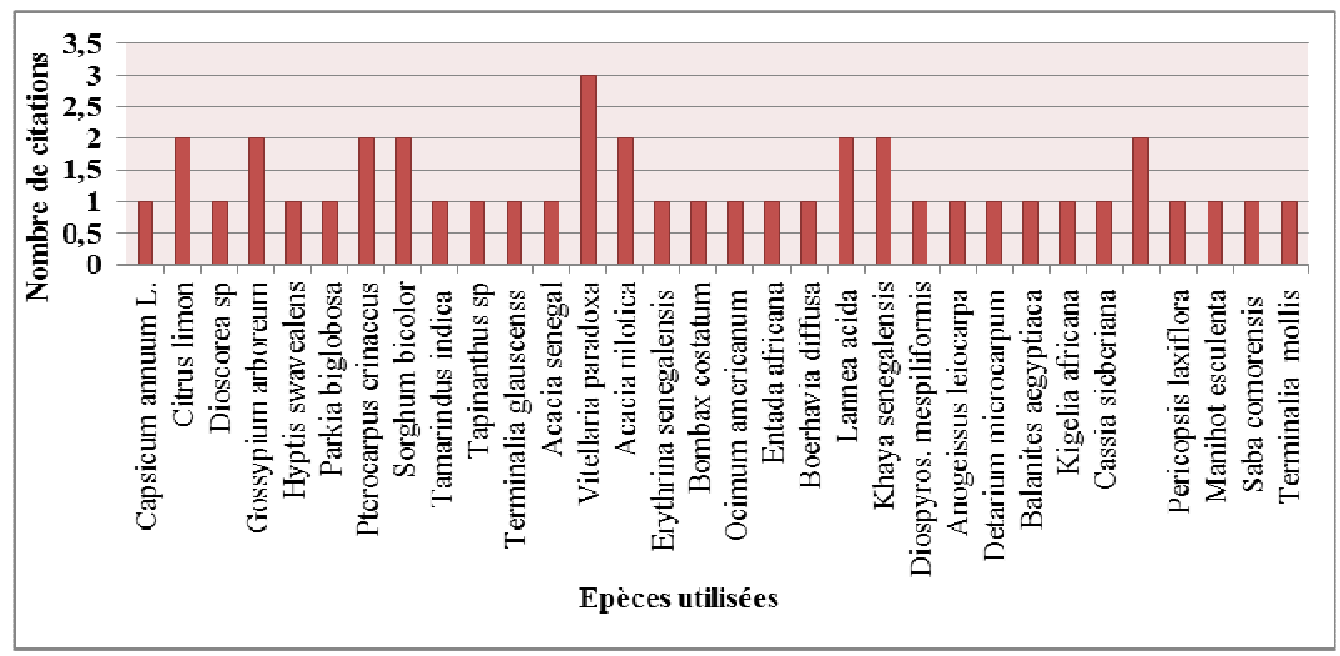

Figure 8 : Fréquence d'usage des plantes identifiées. 
E.M.B. HOUNDJE et al. / Int. J. Biol. Chem. Sci. 10(5): 2090-2107, 2016

Tableau 1 : Inventaire des remèdes thérapeutiques contre la fièvre aphteuse au Bénin.

\begin{tabular}{|c|c|c|c|c|c|c|}
\hline Nom scientifique & $\begin{array}{l}\text { Famille } \\
\text { botanique }\end{array}$ & Noms locaux & Distribution & Organes & $\begin{array}{l}\text { Autres ajouts et } \\
\text { méthode de } \\
\text { préparation }\end{array}$ & $\begin{array}{l}\text { Durée du } \\
\text { traitement }\end{array}$ \\
\hline Capsicum annuиm L. & Solanaceae & Takin(b) & - & Fruit & Macération & Jusqu'à la guérison \\
\hline Citrus limon (L.) Burm.f. & Rutaceae & $\begin{array}{l}\text { Citronnier, limon (fr); } \\
\text { lemon (angl); yovozin, } \\
\text { gbodokle (f, g); } \\
\text { osanorombo }(y, n)\end{array}$ & Ouémé, Couffo, Zou & Feuille. & Poudre + Sel & $7 \mathrm{j}$ \\
\hline \multirow[t]{2}{*}{$\begin{array}{l}\text { Citrus limon ( L.) Burm.f. } \\
+ \\
\text { Dioscorea sp }\end{array}$} & Rutaceae & $\begin{array}{l}\text { yovozin } \\
\text { gbodokle (f, g); } \\
\text { osanorombo }(\mathrm{y}, \mathrm{n})\end{array}$ & Ouémé, Couffo, Zou & Feuille & Poudre & \multirow[t]{2}{*}{$1,5 \mathrm{j}$} \\
\hline & Discoreaceae & $\begin{array}{l}\text { tevi, (f); ewuradudu (y); } \\
\text { dunduhariya (d). }\end{array}$ & & épulchure & Poudre & \\
\hline Gossypium arboreum L. & Malvaceae & $\begin{array}{l}\text { avokanfouncèkè (f); } \\
\text { owoukpikpa (n). }\end{array}$ & $\begin{array}{l}\text { Atlantique, Littoral, } \\
\text { Plateau, Ouémé, } \\
\text { Couffo, Zou, Borgou, } \\
\text { Donga, Atacora, } \\
\text { Alibori }\end{array}$ & Fruit & Macération & $3 \mathrm{j}$ \\
\hline Hyptis suaveolens (L.) Poit. & Lamiaceae & $\begin{array}{l}\text { Hwéflou (f); jogbo, } \\
\text { efinrinaja, kukubi, kutuwi } \\
(y, n) ;\end{array}$ & $\begin{array}{l}\text { Atlantique, Littoral, } \\
\text { Ouémé, Zou }\end{array}$ & Feuille & $\begin{array}{l}\text { Fumigation }+ \text { Fiente de } \\
\text { poule }\end{array}$ & $7 \mathrm{j}$ \\
\hline $\begin{array}{l}\text { Parkia biglobosa (Jacq.) } \\
\text { R.Br. ex Benth. }\end{array}$ & Mimosoideae & $\begin{array}{l}\text { Naréhi }(\mathrm{P}) \text {, ahwatin (f); } \\
\text { ayidanogba }(\mathrm{y}, \mathrm{n}) \text {;suwuru } \\
\text { (b); dààso }(\mathrm{d})\end{array}$ & Ouémé, Atacora & Ecorce & $\begin{array}{l}\text { Macération + Fèces } \\
\text { d'âne }\end{array}$ & $3 \mathrm{j}$ \\
\hline Pterocarpus erinaceus Poir. & Papilionoideae & $\begin{array}{l}\text { teckafricain (fr), barwood } \\
\text { (angl), kozo, (f), osun, }\end{array}$ & $\begin{array}{l}\text { Plateau, Ouémé, } \\
\text { Couffo, Borgou }\end{array}$ & $\begin{array}{l}\text { Sève ou } \\
\text { fruit }\end{array}$ & Frottage & Jusqu'à la guérison \\
\hline
\end{tabular}


E.M.B. HOUNDJE et al. / Int. J. Biol. Chem. Sci. 10(5): 2090-2107, 2016

\begin{tabular}{|c|c|c|c|c|c|c|}
\hline & & $\begin{array}{l}\text { kpakpa, (y, n), kosso } \\
\text { (d).Banouhi (P) }\end{array}$ & & & & \\
\hline $\begin{array}{l}\text { Sorghum bicolor (L.) } \\
\text { Moench }\end{array}$ & Poaceae & $\begin{array}{l}\text { Sorgho (fr); greal millet } \\
\text { (angl); agbokounvovo, } \\
\text { kpokpo (f, g) } \\
\text { Nanwankou(P) }\end{array}$ & $\begin{array}{l}\text { Atlantique, Littoral, } \\
\text { Ouémé, Alibori, } \\
\text { Alibori }\end{array}$ & Graine & Trituration & $\begin{array}{l}\text { 1/j pendant } 1 \\
\text { semaine }\end{array}$ \\
\hline \multirow[t]{2}{*}{ Sorghumsp } & Caesalpinioideae & $\begin{array}{l}\text { Tamarin (fr); larnannd } \\
\text { (angl); jèvivi (f); ajagbàn } \\
(\mathrm{y}, \mathrm{n}) \text {; bobos }(\mathrm{d}) ; \\
\text { Djatami(P) }\end{array}$ & $\begin{array}{l}\text { Sur toute l'étendue } \\
\text { du territoire }\end{array}$ & Tige & \multirow[t]{2}{*}{ Macération } & \multirow[t]{2}{*}{ Jusqu'à la guérison } \\
\hline & Poaceae & $\begin{array}{l}\text { Sorgho, (fr); greal millet } \\
\text { (angl); agbokounvovo, } \\
\text { kpokpo (f, g) }\end{array}$ & $\begin{array}{l}\text { Ouémé, Alibori, } \\
\text { Alibori }\end{array}$ & Tige & & \\
\hline Tapinanthussp & Lythraceae & $\begin{array}{l}\text { Gui africain (fr); } \\
\text { Africanmistletoes (angl); } \\
\text { jodoji (f); oshe, itchè (y, } \\
\text { n); logblaci (a); } \\
\text { daagusuru (b). }\end{array}$ & $\begin{array}{l}\text { Atlantique, Littoral, } \\
\text { Ouémé, }\end{array}$ & Feuille & Poudre + Sel & Jusqu'à la guérison \\
\hline $\begin{array}{l}\text { Terminalia glaucescens } \\
\text { Planch. Ex Benth. } \\
+\end{array}$ & Combretaceae & $\begin{array}{l}\text { Alotun, anagositin (f); } \\
\text { idiodon }(\mathrm{y}, \mathrm{n}) ; \text { aybèrèbu, } \\
\text { bèrokpakiru } \quad(\mathrm{b})\end{array}$ & Couffo, Borgou + & Racine & Macération & $7 \mathrm{j}$ \\
\hline Acacia senegal (L.) Willd. & Mimosoideae & Gommier blanc (fr). & Atacora & écorce & & \\
\hline $\begin{array}{l}\text { Vitellaria paradoxa } \\
\text { C.F.Gaertn.ssp. } \\
\text { paradoxa }\end{array}$ & Scrophulariaceae & $\begin{array}{l}\text { Karité (fr); shea-butter } \\
\text { tree (angl); kotoble, } \\
\text { limutin, wugo }(\mathrm{f}) \text {; tagan } \\
(\mathrm{g}) \text {; akumolapa, èmi, } \\
\text { èmièmi, } \\
\text { èmigidi }(\mathrm{y}, \mathrm{n}), \text { Karéhi }(\mathrm{P})\end{array}$ & $\begin{array}{l}\text { Ouémé, Couffo, Zou, } \\
\text { Borgou, Donga, } \\
\text { Atacora, Alibori }\end{array}$ & écorce & Macération & Jusqu'à la guérison \\
\hline
\end{tabular}


E.M.B. HOUNDJE et al. / Int. J. Biol. Chem. Sci. 10(5): 2090-2107, 2016

\begin{tabular}{|c|c|c|c|c|c|c|}
\hline $\begin{array}{l}\text { Vitellaria paradoxa } \\
\text { C.F.Gaertn.ssp. } \\
\text { paradoxa } \\
+\end{array}$ & $\begin{array}{l}\text { Scrophulariaceae } \\
+\end{array}$ & $\begin{array}{l}\text { Karité (fr); shea-butter } \\
\text { tree (angl); kotoble, } \\
\text { limutin, wugo (f); tagan } \\
(\mathrm{g}) \text {; akumolapa, èmi, } \\
\text { èmièmi, } \\
\text { èmigidi }(\mathrm{y}, \mathrm{n}) \text {, Karéhi }(\mathrm{P})\end{array}$ & $\begin{array}{l}\text { Ouémé, Couffo, Zou, } \\
\text { Borgou, Donga, } \\
\text { Atacora, Alibori }\end{array}$ & $\begin{array}{l}\text { Tige } \\
+\end{array}$ & $\begin{array}{l}\text { Fumigation }+ \text { fiente de } \\
\text { canard et de poule }\end{array}$ & Jusqu'à la guérison \\
\hline Gossypium arboreum L. & Malvaceae & $\begin{array}{l}\text { avokanfouncèkè (f); } \\
\text { owoukpikpa (n). }\end{array}$ & $\begin{array}{l}\text { Atlantique, Littoral, } \\
\text { Plateau, Ouémé, } \\
\text { Couffo, Zou, Borgou, } \\
\text { Donga, Atacora, } \\
\text { Alibori }\end{array}$ & Graine & & \\
\hline $\begin{array}{l}\text { Vitellaria paradoxa } \\
\text { C.F.Gaertn. ssp. } \\
\text { paradoxa+ }\end{array}$ & Scrophulariaceae & $\begin{array}{l}\text { Karité }(\mathrm{fr}) ; \text { shea-butter } \\
\text { tree }(\text { angl); Limutin }(\mathrm{f}) \text {; } \\
\text { tagan }(\mathrm{g}) ; \text { akumolapa, } \\
\text { èmi }(\mathrm{y}, \mathrm{n}) ; \operatorname{Karéhi}(\mathrm{P}) \text {, }\end{array}$ & $\begin{array}{l}\text { Ouémé, Couffo, Zou, } \\
\text { Borgou, Donga, } \\
\text { Atacora, Alibori }\end{array}$ & Ecorce & Décoction & $\begin{array}{l}1 \mathrm{~L} / \mathrm{j} / \text { adulte et } \\
1 / 2 \mathrm{~L} / \mathrm{j} / \text { jeunes } \\
\text { pendant } 3 \text { jours }\end{array}$ \\
\hline $\begin{array}{l}\text { Pterocarpus erinaceus Poir. } \\
+\end{array}$ & Papilionoideae & $\begin{array}{l}\text { teckafricain (fr), barwood } \\
\text { (angl), kozo, (f), osun, } \\
\text { kpakpa, (y, n), kosso } \\
\text { (d).Banouhi (P) }\end{array}$ & $\begin{array}{l}\text { Plateau, Ouémé, } \\
\text { Couffo, Borgou }\end{array}$ & Ecorce & & \\
\hline $\begin{array}{l}\text { Acacia nilotica (L.) Willd. } \\
\text { exDelilessp. Nilotica }\end{array}$ & Mimosoideae & Gadé $(\mathrm{P})$ & $\begin{array}{l}\text { Ouémé, Alibori, } \\
\text { Atacora, Alibori }\end{array}$ & Racine & & \\
\hline $\begin{array}{l}\text { Erythrina senegalensis DC. } \\
+ \\
\text { Bombax costatum Pellegr. }\end{array}$ & Papilionoideae & $\begin{array}{l}\text { Arbre corail (fr), kpaklesi } \\
\text { (f); ologunshèshè, } \\
\text { shokojo }(y, n) .\end{array}$ & $\begin{array}{l}\text { Atlantique, Littoral, } \\
\text { Plateau, Ouémé, } \\
\text { Couffo, Zou, Borgou, } \\
\text { Donga }\end{array}$ & racine & $\begin{array}{l}\text { Macération + Urine de } \\
\text { bœuf }\end{array}$ & $\begin{array}{l}1 / \mathrm{j} \text { Jusqu'à la } \\
\text { guérison }\end{array}$ \\
\hline
\end{tabular}


E.M.B. HOUNDJE et al. / Int. J. Biol. Chem. Sci. 10(5): 2090-2107, 2016

\begin{tabular}{|c|c|c|c|c|c|c|}
\hline & Bombacaceae & $\begin{array}{l}\text { Kapokier à fis rouges de } \\
\text { savane }(\mathrm{fr}) ; \text { huntin }(\mathrm{f}) ; \\
\text { tarago }(\mathrm{g}) ; \text { ponpola }(\mathrm{y}, \mathrm{n}) \text {; } \\
\text { forgo }(\mathrm{d}), \text { Kourouhi }(\mathrm{P})\end{array}$ & Zou, Borgou & & & \\
\hline Ocimum americanum $\mathrm{L}$. & Lamiaceae & $\begin{array}{l}\text { Basilic blanc (fr); } \\
\text { kesukesu, xisixisi (f); } \\
\text { haciyayo (g); efinrinata, } \\
\text { aruntani tan (y, } \\
\text { n); nyàka (b) }\end{array}$ & $\begin{array}{l}\text { Zou, Alibori, un peu } \\
\text { partout en faible } \\
\text { proportion }\end{array}$ & Feuille & Trituration + Potasse & $\begin{array}{l}\text { 2/j Jusqu'à la } \\
\text { guérison }\end{array}$ \\
\hline $\begin{array}{l}\text { Entada africana Guill. } \\
\text { \&Perr. }\end{array}$ & Mimosaceae & $\begin{array}{l}\text { Néré des éléphants }(\mathrm{fr}) ; \\
\text { kake }(\mathrm{f}) ; \text { ogurobe, } \\
\text { akakanyi, akawayi }(\mathrm{y}, \mathrm{n}) ; \\
\text { worudoru (ba); } \\
\text { menarunyim }(\mathrm{d}) \text {, } \\
\text { Toufalderehi }(\mathrm{P})\end{array}$ & $\begin{array}{l}\text { Couffo, } \\
\text { Borgou,Donga }\end{array}$ & Ecorce & Décoction + Potasse & $\begin{array}{l}\text { 1/j Jusqu'à la } \\
\text { guérison }\end{array}$ \\
\hline Boerhavia diffusa $\mathrm{L}$. & Nyctaginaceae & $\begin{array}{l}\text { Herbe cochon }(\mathrm{fr}) ; \\
\text { handoukpo (f); etikpéléla. } \\
\text { tinkpingnila }(\mathrm{y}, \mathrm{n}) ; \\
\text { twébissou }(\mathrm{b}) \\
\text { Gbagbanati }(\mathrm{P})\end{array}$ & $\begin{array}{l}\text { Atlantique, Littoral, } \\
\text { Ouémé, Alibori }\end{array}$ & Ecorce & Décoction + Potasse & $\begin{array}{l}\text { 1/j Jusqu'à la } \\
\text { guérison }\end{array}$ \\
\hline $\begin{array}{l}\text { Acacia nilotica } \\
\text { Willd. ex Delile ssp. Nilotica }\end{array}$ & Mimosaceae & Gadé(p) & $\begin{array}{l}\text { Ouémé, Alibori, } \\
\text { Atacora, Alibori }\end{array}$ & $\begin{array}{l}\text { Fruit ou } \\
\text { écorce }\end{array}$ & Macération + Urine & $2 / \mathrm{j}$ pendant 1 mois \\
\hline Lannea acida A.Rich. s.l. & Anacardiaceae & $\begin{array}{l}\text { Tchami1, Zonzon (f); } \\
\text { akunu, akuhu (y, n); } \\
\text { yadibu (ba); taykoudako } \\
\text { (d). oganwo, aganwo (y, } \\
\text { n); felè (d) }\end{array}$ & $\begin{array}{l}\text { Couffo, Borgou, } \\
\text { Alibori, Donga, } \\
\text { Atacora, Alibori }\end{array}$ & Racine & $\begin{array}{l}\text { Calcination }+ \text { œuf de } \\
\text { poule }+ \text { sel }\end{array}$ & $\begin{array}{l}\text { 1/j pendant } 2 \\
\text { semaines }\end{array}$ \\
\hline
\end{tabular}


E.M.B. HOUNDJE et al. / Int. J. Biol. Chem. Sci. 10(5): 2090-2107, 2016

\begin{tabular}{|c|c|c|c|c|c|c|}
\hline $\begin{array}{l}\text { Lannea acida A.Rich. s.l. } \\
+\end{array}$ & Anacardiaceae & $\begin{array}{l}\text { Tchami (P), Zonzon (f); } \\
\text { akunu, akuhu (y, n); } \\
\text { yadibu (b); taykoudako } \\
\text { (d). }\end{array}$ & $\begin{array}{l}\text { Couffo, Borgou, } \\
\text { Alibori, Donga, } \\
\text { Atacora, Alibori }\end{array}$ & & & \\
\hline $\begin{array}{l}\text { Khaya senegalensis ( Desr.) } \\
\text { A.Juss. } \\
+\end{array}$ & Meliaceae & $\begin{array}{l}\text { Caïlcédrat (fr); Senegal } \\
\text { mahogany (angl); } \\
\text { zunzatin (f); agawu, (g); } \\
\text { oganwo, ago, (y, n); felè } \\
(d) ; \text { Kahi }(P)\end{array}$ & Donga & Ecorce & Infusion & $\begin{array}{l}1 / \mathrm{j} \text { pendant } \\
\text { unesemaine }\end{array}$ \\
\hline $\begin{array}{l}\text { Diospyros mespiliformis } \\
\text { Hochst. ex } \\
\text { A.DC. }\end{array}$ & Ebenaceae & $\begin{array}{l}\text { Goyavier du singe (fr); } \\
\text { Ken (f); kanran, ijidudu } \\
(\mathrm{y}, \mathrm{n}) \text {; wonyibu (b); tuuri } \\
(\mathrm{d}) \text {; Ganeï (P) }\end{array}$ & $\begin{array}{l}\text { Ouémé, Couffo, Zou, } \\
\text { Borgou, Alibori, } \\
\text { Atacora }\end{array}$ & & & \\
\hline $\begin{array}{l}\text { Anogeis susleiocarpa (De.) } \\
\text { GuUl. \&Perr. }\end{array}$ & Combretaceae & $\begin{array}{l}\text { Hlihon, (f); anyi ma, ayin } \\
(\mathrm{y}, \mathrm{n}) \text {; halekete (b); } \\
\text { agbaouna (d), Kodjoli } \\
\text { (P) }\end{array}$ & $\begin{array}{l}\text { Ouémé, Couffo, Zou, } \\
\text { Borgou, }\end{array}$ & Ecorce & Poudre & $\begin{array}{l}\text { Tous les } 2 \mathrm{j} \\
\text { pendant une } \\
\text { semaine }\end{array}$ \\
\hline $\begin{array}{l}\text { Detarium microcarpum } \\
\text { Guill. \&Perr. }\end{array}$ & Caesalpinioideae & $\begin{array}{l}\text { Petit détar (fr); tallowtree } \\
\text { (angl); iyede (y); Konkéhi } \\
\text { (P) }\end{array}$ & $\begin{array}{l}\text { Ouémé, Couffo, } \\
\text { Borgou, Alibori }\end{array}$ & Feuille & Trituration & $1 / \mathrm{j}$ pendant $3 \mathrm{j}$ \\
\hline $\begin{array}{l}\text { Balanites aegyptiaca (L.) } \\
\text { Delile }\end{array}$ & Balanitaceae & $\begin{array}{l}\text { Dattierégyptien (fr); Soap } \\
\text { berry tree (angl); } \\
\text { egungunekun,budaré (y, } \\
\text { n); gamèlè (b); garbè }(\mathrm{d}) \text {; } \\
\text { Tané }(\mathrm{P}) \text {, }\end{array}$ & $\begin{array}{l}\text { Alibori, Donga, } \\
\text { Atacora, Alibori }\end{array}$ & Ecorce & Poudre + Sel & $\begin{array}{l}\text { 3/j jusqu'à la } \\
\text { guérison }\end{array}$ \\
\hline Kigelia Africana (Lam.) Benth. & Bignoniaceae & $\begin{array}{l}\text { Faux baobab (fr); } \\
\text { cucumber tree (angl); anon } \\
\text { anon (f); anyakpo }\end{array}$ & $\begin{array}{l}\text { Atlantique, Littoral, } \\
\text { Ouémé, Couffo, Zou, } \\
\text { Borgou }\end{array}$ & Fruit & Décoction & $\begin{array}{l}1 / 2 \mathrm{~L} / \mathrm{j} \text { par animal } \\
\text { pendant } 3 \mathrm{j}\end{array}$ \\
\hline
\end{tabular}


E.M.B. HOUNDJE et al. / Int. J. Biol. Chem. Sci. 10(5): 2090-2107, 2016

\begin{tabular}{|c|c|c|c|c|c|c|}
\hline & & $\begin{array}{l}\text { (g); kpandoro, uya }(\mathrm{y}, \mathrm{n}) \text {; } \\
\text { Djilidjalahi }(\mathrm{P})\end{array}$ & & & & \\
\hline Cassia sieberiana DC. & Caesalpinioideae & $\begin{array}{l}\text { Cassia de Sieber (fr); (West) } \\
\text { African labumum (angl); } \\
\text { kohi(P) }\end{array}$ & $\begin{array}{l}\text { Atlantique, Littoral, } \\
\text { Plateau, Couffo, } \\
\text { Borgou, Alibori, Donga }\end{array}$ & Ecorce & Décoction & $1 / \mathrm{j}$ pendant $3 \mathrm{j}$ \\
\hline $\begin{array}{l}\text { Agelanthus dodoneifolius (DC.) } \\
\text { Po1h. \&Wiens }\end{array}$ & Loranthaceae & Sôtô (P) & $\begin{array}{l}\text { Plateau, Couffo, Zou, } \\
\text { Borgou, Alibori, Donga }\end{array}$ & Feuille & Fumigation & $1 / \mathrm{j}$ pendant $2 \mathrm{j}$ \\
\hline $\begin{array}{l}\text { Pericopsis laxiflora } \text { (Benth. ex } \\
\text { Baker) } \\
\text { Meeuwen }\end{array}$ & Papilionoideae & $\begin{array}{l}\text { Faux dalbergia (fr), sèdon } \\
\text { (f), shèdon, ishedu }(y, n) . ; \\
\text { Cinanfégou2 ou } \\
\text { sorokouhi1 }\end{array}$ & $\begin{array}{l}\text { Ouémé, Couffo, Zou, } \\
\text { Borgou, Alibori, } \\
\text { Atacora }\end{array}$ & Ecorce & Poudre + Sel & $\begin{array}{l}\text { 1/semaine jusqu'à la } \\
\text { guérison }\end{array}$ \\
\hline Manihotes culenta Crantz & Caesalpinioideae & $\begin{array}{l}\text { Manioc (fr); cassava (angl); } \\
\text { fenyen (f); fenyen } \\
\text { (g); paki, gbaguda (y, n); } \\
\text { logo (ba), loogo (d); Loogoh } \\
\text { (p) }\end{array}$ & $\begin{array}{l}\text { Cotier, Plateau, Ouémé, } \\
\text { Couffo, Zou, Borgou, } \\
\text { Alibori, }\end{array}$ & Feuille & Poudre + fèces de caprin & $1 / \mathrm{j}$ pendant 1 mois \\
\hline $\begin{array}{l}\text { Saba comorensis (Boj.) Pichon } \\
+\end{array}$ & Apocynaceae & $\begin{array}{l}\text { Ibo, gidigidi (y, n); loo } \\
\text { (d) ; Bênahi (p) }\end{array}$ & $\begin{array}{l}\text { Couffo, Zou, Borgou, } \\
\text { Alibori }\end{array}$ & Ecorce & \multirow[t]{2}{*}{ Trituration + oeuf de poule } & \multirow{2}{*}{$\begin{array}{l}3 / \mathrm{j} \text { jusqu'à la } \\
\text { guérison }\end{array}$} \\
\hline $\begin{array}{l}\text { Agelanthus dodoneifolius (DC.) } \\
\text { Po1h. \&Wiens }\end{array}$ & Loranthaceae & $\begin{array}{l}\text { Comme des Tapinanthus ; } \\
\text { Sôtô (P) }\end{array}$ & $\begin{array}{l}\text { Plateau, Couffo, Zou, } \\
\text { Borgou, Alibori, Donga }\end{array}$ & Feuille & & \\
\hline $\begin{array}{l}\text { Khaya senegalensis (Desr.) } \\
\text { A.Juss. }\end{array}$ & Meliaceae & $\begin{array}{l}\text { Caïlcédrat (fr); Senegal } \\
\text { mahogany (angl); zunzatin } \\
\text { (f); agawu, (g); oganwo, } \\
\text { ago, }(\mathrm{y}, \mathrm{n}) \text {; felè }(\mathrm{d}) ; \text { Kahi }(\mathrm{P})\end{array}$ & Donga & Ecorce & Macération + urine de boeuf & $\begin{array}{l}2 / \mathrm{j} \text { pendant } \\
\text { unesemaine }\end{array}$ \\
\hline $\begin{array}{l}\text { Termina liamollis } \\
\text { M.A.Lawson }\end{array}$ & Combretaceae & Tiguerehi $(\mathrm{P})$ & Borgou, Atacora & $\begin{array}{l}\text { Ecorce }+ \\
\text { racine }\end{array}$ & Décoction & $2 / \mathrm{j}$ pendant 3 jours \\
\hline
\end{tabular}




\section{DISCUSSION}

Les résultats obtenus au cours de cette étude ont montré que la totalité des enquêtés est de sexe masculin. Cela confirme ceux obtenus par Kaboré et al. (2007) qui indiquent qu'en Afrique, très rares sont les femmes propriétaires de troupeaux bovins. Ce constat est contraire à ceux faits par Youssao et al. (2013) dans le département du Borgou où il y avait quelques femmes propriétaires. L'absence ou la faible implication des femmes dans la gestion des élevages de grands ruminants explique bien le fait que les connaissances ethno-vétérinaires sont beaucoup plus l'apanage des individus de sexe masculin. La majorité des éleveurs rencontrés et pratiquant la médecine traditionnelle vétérinaire à base de plantes sont dans la tranche d'âge de 30 à 60 ans. Ceci montre que les connaissances ethno-vétérinaires sont détenues par les personnes âgées mais aussi transmises de plus en plus aux jeunes générations en charge du suivi des animaux (Klotoé et al., 2013). Les éleveurs enquêtés sont majoritairement des peulhs et bon nombre d'entre eux ont un niveau d'instruction faible indiquant ainsi que les savoirs traditionnels ne sont pas en corrélation avec l'instruction d'un individu. De ce fait comme l'a souligné Ogni (2016), l'absence d'instruction d'un individu n'affecte aucunement son savoir endogène. Dans la majorité des élevages parcourus, les éleveurs sont majoritairement d'ethnie peulh. Ce résultat corrobore ceux obtenus par Dassou et al. (2014), Ogni et al. (2014) et Kpodékon et al. (2015a) qui ont également mené des enquêtes ethno-pharmacologiques sur l'ensemble du territoire béninois.

La reconnaissance de la fièvre aphteuse est généralement établie par tous les éleveurs à travers l'un de ses signes. C'est une maladie endémique qui sévit tous les mois dans un département ou un autre du pays selon les rapports de la Direction de l'élevage. La fièvre aphteuse est d'autant plus présente qu'elle constitue la pathologie qui sévit le plus dans les troupeaux (Dassou et al., 2014). La variation des périodes favorables d'apparition de la pathologie est certainement due à la présence d'un des facteurs favorisant les déplacements des animaux que sont le pâturage, l'eau et parfois les marchés à bétail (Rweyemamu et al., 2008).

Ainsi, les troupeaux se retrouvent à un moment de l'année dans le département qui leur est propice pour l'alimentation et l'abreuvement. Cette pratique a été également révélée par les études de Couacy-Hymann et al. (2006) qui a pu tracer l'itinéraire suivi par les troupeaux au Bénin lors de leur déplacement. La quasi-totalité des éleveurs enquêtés font recours aussi bien à la médecine moderne que traditionnelle. Les résultats de l'enquête ont révélé qu'avant l'arrivée du vétérinaire, les premiers soins administrés aux animaux sont à base de plantes médicinales. Le traitement des plaies se fait à base d'antibiotique. Les mêmes constats ont été faits par plusieurs auteurs à travers leurs études faites au nord du Bénin sur les systèmes d'élevage (Youssao et al., 2013; Yacoubou, 2011 ; Alkoiret et al., 2009). Les soins ethno-vétérinaires se font en amont et aval c'est à dire que l'éleveur associe les soins vétérinaire et traditionnel pour traiter ses animaux.

A travers cette étude, différentes recettes ethno-vétérinaires à base de plantes ont été recensées pour le traitement de la fièvre aphteuse. Au vue des recettes obtenues, l'espèce Vitellaria paradoxa est celle qui a été plus citée par les éleveurs. Cela confirme ainsi les observations faites par Dassou et al. (2014) qui indiquent l'usage confirmée de Vitellaria paradoxa par les éleveurs dans le traitement de la fièvre aphteuse à cause de son pouvoir cicatrisant. Dans le Nord de la Côte d'Ivoire, les éleveurs utilisent cette plante pour traiter la diarrhée sanguinolente chez les animaux (Koné et Kamazi, 2008). Au Cameroun, dans la région de Bénoué par contre, la macération de l'écorce de Vitellaria paradoxa est utilisée contre les pathologies gastro-intestinales chez les ruminants (Djoueche et al., 2011). D'autres espèces végétales recensées au cours de cette étude ont été aussi utilisées pour traiter d'autres 
pathologies animales. Ainsi, on a par exemple les espèces telles que Khaya senegalensis et Kigelia africana utilisées dans le traitement de la trypanosome (Ogni et al., 2014) ou Lannea acida utilisée pour la maladie du Newcastle. De ce fait, la même plante peut être utilisée pour traiter plusieurs pathologies.

Les parties de plantes qui interviennent le plus dans les recettes sont respectivement l'écorce et les feuilles comme l'ont montré Dassou et al. (2014). La macération est le mode de préparation des plantes le plus utilisé. Chez les plantes galactogènes, la consommation directe de fruits ou à l'application locale sur les parties lésées est la plus fréquente (Akouedegni et al., 2012). Il est surtout important de noter que le mode de préparation ainsi que les parties à utiliser de la plante dépendent du type de pathologies à traiter.

A partir de cette étude et celles de Byavu et al. (2000) ; Kaboré et al. (2007), il est à retenir que les motivations des éleveurs sont les mêmes en ce qui concerne l'usage des plantes pour le traitement des animaux.

Parmi les 32 plantes obtenues au cours de cette étude, 8 espèces ont été identifiées par les études de Dassou et al. (2014) et 23 espèces chez Kpodékon et al. (2015b) pour le traitement de la fièvre aphteuse. Ces mêmes espèces sont également utilisées pour contrôler la peste des petits ruminants, la variole aviaire, la peste porcine africaine et la maladie de Newcastle. Cette augmentation du nombre d'espèces utilisées dans le traitement pour la fièvre aphteuse est due au fait que la présente a été effectuée presque sur toute l'étendue du territoire national et est exclusivement axée sur le contrôle de ladite pathologie. En effet, seul le département du Littoral a été exclu à cause de son statut purement urbain.

\section{Conclusion}

L'inventaire des connaissances actuelles sur les plantes médicinales indique qu'au Bénin, 32 plantes sont utilisées dans le traitement de la fièvre aphteuse. Cette étude revêt une importance capitale dans la mesure où dans le contexte actuel la maladie sévit au Bénin tout au long de l'année. Les recherches de vaccins contre la fièvre aphteuse dans ce pays sont encore en cours et l'utilisation appropriée des plantes pourrait être un premier recours pour l'éleveur en l'absence de mesures sanitaires. Les quantités exactes d'organes de plantes à prélever ainsi que des différentes composantes des recettes sont autant d'éléments sur lesquels des études peuvent porter. Aussi il serait important d'effectuer de tests cliniques et de laboratoire pour évaluer l'effet thérapeutique réel des espèces répertoriées.

\section{CONFLIT D'INTERETS}

Les auteurs ne déclarent aucun conflit d'intérêt.

\section{CONTRIBUTION DES AUTEURS}

TMK: validation du protocole de recherche, suivi de la collecte des données, instructions pour la rédaction et la correction du manuscrit; EMH: rédaction du protocole, collecte des données et rédaction du manuscrit; CAO et NN : appui à la collecte des données et à l'identification des plantes; AKIY et SF: validation des données analysées et lecture du manuscrit.

\section{REMERCIEMENTS}

Les auteurs remercient Dassou Hospice pour sa participation à l'identification de certaines plantes. Ils adressent également leur gratitude à la Direction de l'Elevage et aux éleveurs qui ont contribué à la réalisation de l'étude.

\section{REFERENCES}

Akouedegni CG, GbégoTossa I, Daga FD, Koudandé DO, Hounzangbé-Adoté MS. 2012. Synthèse des connaissances sur les plantes galactogènes et leurs usages en République du Bénin. Bul. Rec. Agr. Bénin, Numéro spécial: 24-35.

Alkoiret IT, Awohouedji DYG, Akossou AYJ, Bosma RH. 2009. Typologie des systèmes d'élevage bovin de la commune de Gogounou au Nord-Est du 
Bénin. Ann. Sci. Agro. Bénin, 2(12): 7798.

Byavu N, Henrard C, Dubois M, Malaisse F. 2000. Phytothérapie traditionnelle des bovins dans les élevages de la plaine de la Ruzizi. Biotechnol. Agron. Soc. Environ., 4(3): 135-156.

Couacy-Hymann E, Aplogan GL, Sangare O, Compaore Z, Karimu J, Awouémé KA, Seini A, Martin V, Valarcher JF. 2006. Etude rétrospective de la Fièvre Aphteuse en Afrique de l'Ouest de 1970 à 2003. Rev. Sci. Tech. Off. Int. Epiz., 25(3) : 1013-1024.

Dassou HG, Ogni CA, Yédomonhan $\mathrm{H}$, Adomou AC, Tossou M, Dougnon JT, Akoègninou A. 2014. Diversité, usages vétérinaires et vulnérabilité des plantes médicinales au Nord-Bénin. Int. J. Biol. Chem. Sci., 8(1): 189-210.

Schönegg G, Martel P, Sano B, Noufou S, Zeh M. 2006. Les Conflits liés à la Transhumance Transfrontalière entre le Niger, le Burkina Faso et le Bénin. ( $1^{\text {ère }}$ ed). DED Press: Berlin.

Djoueche CM, Azebaze AB, Dongmo AB. 2011. Investigation of plants used for the ethnoveterinary control of gastrointestinal parasites in Bénoué region, Cameroon. TROP, 29(4): 205211.

Fasina FO, Connell DR, Talabi OA, Lazarus DD, Adeleke GA, Olusanya TP, Hernandez JA. 2013. Foot-and-mouth disease virus strains and examination of exposure factors associated with seropositivity of cattle herds in Nigeria during 2007-2009. Prev. Vet. Med., 109 (3-4): 334-42.

Gorna K, Houndjè E, Romey A, Relmy A, Blaise-Boisseau S, Kpodékon $\mathrm{M}$, Saegerman C, Moutou F, Zientara S, Bakkali L. 2014. First isolation and molecular characterization of foot-andmouth disease virus in Benin. Vet. Micr., 171 : 175-181.

Grubman MJ, Baxt B. 2004. Foot and mouth disease. Clin. Microbiol. Rev., 17: 465493.
Kaboré A, Tamboura HH, Belem GAM, Traoré A. 2007. Traitements ethnovétérinaire des parasitoses digestives des petits ruminants dans le plateau central du Burkina Faso. Int. J. Biol. Chem. Sci., 1(3): 297-304.

Klotoé JR, Dougnon TV, Koudouvo K, Atègbo JM, Loko $\mathrm{F}$, Akoègninou $\mathrm{A}$, Aklikokou K, Dramane K, Gbeassor M. 2013. Ethnopharmacological survey on antihemorrhagic medicinal plants in South of Benin. European J. Med. Plants, 3(1): 40-51.

Koné WM, Kamanzi Atindehou K. 2008. Ethnobotanical inventory of medicinal plants used in traditional veterinary medicine in Northern Côte d'Ivoire (West Africa). S. Afr. J. Bot., 74(1): 7684.

Kpodekon TM, Dotche OI, Youssao Abdou Karim I, Noudeke N, Farougou S. 2015a. Inventaire des plantes utilisées pour le contrôle des maladies à incidence négative sur la production du lait. Actes du Symposium de Pathologie et de Santé Animales dans les pays de l'espace de l'Union Economique et Monétaire Ouest Africaine (UEMOA). Université d'Abomey-Calavi, 02 et 03 décembre 2015, Bénin.

Kpodékon TM, Ogni CA, Dassou H, Dougnon TJ, Boko C, Koutinhouin GB, Goussanou JSE, Akoegninou A, Youssao I. 2015b. Dominant viral pathologies in the extensive and semiintensive animal breeding and their treatment mode in ethno-veterinary medicine in Benin. Vet. World, 8(12): 1424-1434.

INSAE. 2004. Cahier des villages et quartiers de ville Troisième Recensement Général de la population et de l'habitat, p. 23.

Ogni CA, Kpodekon MT, Dassou HG, Boko CK, Koutinhouin BG, Dougnon JT, Youssao AKI, Yedomonhan H, Akoegninou A. 2014. Inventaire ethnopharmacologique des plantes utilisées dans le traitement des pathologies parasitaires dans les élevages extensifs et 
semi-intensifs du Bénin. Int. J. Biol. Chem. Sci., 8(3): 1089-1102.

Ogni CA, Kpodekon M, Dougnon J, Dassou H, Goussanou JE, Boko C, Koutinhouin B, Youssao I, Akoegninou A. 2016. Dominant bacterial diseases in the extensive and semi-intensive animal breeding and their treatment method by ethnoveterinary medicine in Benin. $J$. App. Pharm. Sci., 6(04): 150-158.

Ogni CASW. 2016. Enquête ethnopharmacologique et efficacité des extraits de quelques plantes sur deux pathologies animales fréquentes au Bénin. Thèse de doctorat, Université d'Abomey-Calavi, Bénin, p. 215.

Rweyemamu MP, Mackay D, Sumption K, Brownlie J, Leforban Y, Valarcher J-F, Knowles NJ, Saraiva V. 2008. Epidemiological patterns of foot and mouth disease worldwide. Transbound. Emerg. Dis, 55 : 57-72.
Toyang NJ, Wanyama J, Nuwanyakpa M, Django S. 2007. Ethnomédecine Vétérinaire: une Approche Pratique du Traitement des Maladies du Bétail en Afrique Subsaharienne ( $1^{\text {èr }}$ edn). Agrodok: Wageningen.

Yacoubou AS. 2011. Suivi sanitaire des bovins de race Borgou : cas des Communes de Sinendé et de OuassaPéhunco. Mémoire de fin de formation pour l'obtention du diplôme de Licence Professionnelle en Production Animale, Ecole Polytechnique d'Abomey-Calavi, p. 55.

Youssao AKI, Dahouda M, Attakpa EY, Koutinhouin GB, Ahounou GS, Toleba SS, Balogoun BS. 2013. Diversité des systèmes d'élevages de bovins de race bovine Borgou dans la zone soudanienne du Bénin. Int. J. Biol. Chem. Sci., 7(1): 125-146. 